этом необходимо сконцентрировать внимание на развитие технологий обеспечения безопасности данных.

Таким образом, организация эффективного использования цифровых технологий с учетом возможностей банка и запросов со стороны клиентов будут основными направлениями для цифровизации банковского сектора в ближайшие годы.

Благодаря удобству использования инновационных продуктов, интерес к которым за последние годы только повышается, цифровые технологии обладают огромным потенциалом развития не только для банков, но и для экономики в целом. Текущее состояние банковского сектора России предоставляет все необходимые предпосылки для дальнейшей его цифровизации и будущего мирового лидерства в этой сфере. Рациональные цифровые преобразования позволят отдельным банкам, а впоследствии и всему банковскому сектору повысить эффективность деятельности и выйти на новый этап развития финансово-кредитных организаций.

$$
* * *
$$

1. Миронова Д.Д., Трудкова Е.В. Интернет-банкинг как инновационная технология банковского обслуживания // В сборнике: Научная весна-2018. Экономические науки: Сборник научных трудов. Научное электронное издание. Шахты, 2018. С. 19-22.

2. Черкесова Э.Ю., Миронова Д.Д., Трудкова Е.В. Биометрия как один из основных показателей банковской безопасности // Научная весна-2019. Экономические науки: Сборник научных трудов. Научное электронное издание. Шахты, 2019. С. 192-197.

3. Черкесова Э.Ю., Миронова Д.Д. Состояние и перспективы цифровой трансформации экономики в Российской Федерации // Экономика и предпринимательство. 2019. № 6 (107). С. 107-114.

4. НАФИ назвал самые популярные среди россиян цифровые сервисы банкв [Электронный ресурс]. URL: https://www.kommersant.ru/doc/4157220 (дата обращения: 15.04.2021).

5. Цифровизация услуг в России: уже на пороге [Электронный ресурс]. URL: https://wciom.ru/analytical-reviews/analiticheskii obzor/czifrovizacziya-uslug-v-rossii-uzhe-na-poroge(дата обращения: 18.04.2021)

\title{
Куренков Д.А. \\ Разработка сервиса для решения задач маршрутизации обращений пользователей в службу поддержки с помощью методов машинного обучения
}

Национальный исследовательский ядерный университет «МИФИ» doi: $10.18411 / \mathrm{j}-05-2021-15$

(Россия, Москва)

\section{Аннотация}

Проблема поддержки ИТ-инфраструктуры - механическое реагирование и распределение запросов пользователей. А главная стратегическая цель управления ИТсредой заключается в повышении качества обслуживания пользователей.

Основную часть информации о внешнем мире человек получает по зрительному каналу и далее весьма эффективно обрабатывает полученную информацию при помощи аппарата анализа и интерпретации информации. Поэтому встает вопрос о возможности машинной реализации данного процесса, которую возможно решить с помощью метода автоматического анализа данных и методов машинного обучения.

Ключевые слова: машинное обучение, механическое реагирование, автоматизация процессов, пользователь, обращание.

\section{Abstract}

The problem of IT infrastructure support is mechanical response and distribution of user requests. And the main strategic goal of managing the IT environment is to improve the quality of user experience.

A person receives the main part about the external world through the visual channel and effectively processes the information received by analyzing and interpreting information. 
Therefore, the question arises about the possibility of machine implementation of this process, which can be solved using the method of automatic data analysis and machine learning methods.

Key words: machine learning, mechanical response, process automation, user, handling.

\section{1. Изучение метода автоматического анализа данных}

Деревья решений являются одним из наиболее эффективных инструментов интеллектуального анализа данных и предсказательной аналитики, которые позволяют решать задачи классификации и регрессии.

Они представляют собой иерархические древовидные структуры, состоящие из решающих правил вида «Если ..., то ...». Правила автоматически генерируются в процессе обучения на обучающем множестве и, поскольку они формулируются практически на естественном языке, деревья решений как аналитические модели более вербализуемы и интерпретируемы, чем, скажем, нейронные сети.

Дерево решений - это метод представления решающих правил в иерархической структуре, состоящей из элементов двух типов - узлов и листьев. В узлах находятся решающие правила и производится проверка соответствия примеров этому правилу по какому-либо атрибуту обучающего множества.

В простейшем случае, в результате проверки, множество примеров, попавших в узел, разбивается на два подмножества, в одно из которых попадают примеры, удовлетворяющие правилу, а в другое - не удовлетворяющие.

Затем к каждому подмножеству вновь применяется правило и процедура рекурсивно повторяется пока не будет достигнуто некоторое условие остановки алгоритма. В результате в последнем узле проверка и разбиение не производится и он объявляется листом. Лист определяет решение для каждого попавшего в него примера. Для дерева классификации - это класс, ассоциируемый с узлом, а для дерева регрессии - соответствующий листу модальный интервал целевой переменной.

Таким образом, в отличие от узла, в листе содержится не правило, а подмножество объектов, удовлетворяющих всем правилам ветви, которая заканчивается данным листом.

Основная сфера применения деревьев решений - поддержка процессов принятия управленческих решений, используемая в статистике, анализе данных имашинном обучении. Задачами, решаемыми с помощью данного аппарата, являются:

- Классификация- отнесение объектов к одному из заранее известных классов. Целевая переменная должна иметь дискретные значения.

- Регрессия (численное предсказание) - предсказание числового значения независимой переменной для заданного входного вектора.

- Описание объектов — набор правил в дереве решений позволяет компактно описывать объекты. Поэтому вместо сложных структур, описывающих объекты, можно хранить деревья решений.

Процесс построения деревьев решений заключается в последовательном, рекурсивном разбиении обучающего множества на подмножества с применением решающих правил в узлах. Процесс разбиения продолжается до тех пор, пока все узлы в конце всех ветвей не будут объявлены листьями. Объявление узла листом может произойти естественным образом (когда он будет содержать единственный объект, или объекты только одного класса), или по достижении некоторого условия остановки, задаваемого пользователем (например, минимально допустимое число примеров в узле или максимальная глубина дерева).

Алгоритмы построения деревьев решений относят к категории так называемых жадных алгоритмов. Жадными называются алгоритмы, которые допускают, что локально-оптимальные решения на каждом шаге (разбиения в узлах), приводят к 
оптимальному итоговому решению. В случае деревьев решений это означает, что если один раз был выбран атрибут, и по нему было произведено разбиение на подмножества, то алгоритм не может вернуться назад и выбрать другой атрибут, который дал бы лучшее итоговое разбиение. Поэтому на этапе построения нельзя сказать обеспечит ли выбранный атрибут, в конечном итоге, оптимальное разбиение.

В основе большинства популярных алгоритмов обучения деревьев решений лежит принцип «разделяй и властвуй». Алгоритмически этот принцип реализуется следующим образом. Пусть задано обучающее множество S, содержащее nn примеров, для каждого из которых задана метка класса $\mathrm{Ci}(\mathrm{i}=1 . . \mathrm{k})$, и $\mathrm{m}$ атрибутов $\mathrm{Aj}(\mathrm{j}=1 . . \mathrm{m})$, которые, как предполагается, определяют принадлежность объекта к тому или иному классу. Тогда возможны три случая:

- Bce примеры множества S имеют одинаковую метку класса Ci (т.е. все обучающие примеры относятся только к одному классу). Очевидно, что обучение в этом случае не имеет смысла, поскольку все примеры, предъявляемые модели, будут одного класса, который и «научится» распознавать модель. Само дерево решений в этом случае будет представлять собой лист, ассоциированный с классомСі. Практическое использование такого дерева бессмысленно, поскольку любой новый объект оно будет относить только к этому классу.

- Множество S вообще не содержит примеров, т.е. является пустым множеством. В этом случае для него тоже будет создан лист (применять правило, чтобы создать узел, к пустому множеству бессмысленно), класс которого будет выбран из другого множества (например, класс, который наиболее часто встречается в родительском множестве).

- Множество S содержит обучающие примеры всех классов Ck. В этом случае требуется разбить множество $\mathrm{S}$ на подмножества, ассоциированные с классами. Для этого выбирается один из атрибутов $\mathrm{Aj}$ множества $\mathrm{S}$ который содержит два и более уникальных значения (a1,a2,..,ap), где $\mathrm{p}$ - число уникальных значений признака. Затем множество $\mathrm{S}$ разбивается на рр подмножеств $(\mathrm{S} 1, \mathrm{~S} 2, \ldots, \mathrm{Sp})$, каждое из которых включает примеры, содержащие соответствующее значение атрибута. Затем выбирается следующий атрибут и разбиение повторяется. Это процедура будет рекурсивно повторяться до тех пор, пока все примеры в результирующих подмножествах не окажутся одного класса.

\section{принятия решений \\ 2. Исследование классификации и регрессии с помощью деревьев}

В целом, анализ дерева решений - это инструмент прогнозного моделирования, который можно применять во многих областях. Деревья решений могут быть построены с помощью алгоритмического подхода, который может разбить набор данных по-разному в зависимости от условий. Решения Tress являются наиболее мощными алгоритмами, которые подпадают под категорию контролируемых алгоритмов.

Их можно использовать как для задач классификации, так и для регрессии. Две основные сущности дерева - это узлы принятия решений, где данные разделяются и удаляются, где мы получаем результат.

Дерево решений - это контролируемая модель машинного обучения, используемая для прогнозирования цели путем изучения правил принятия решений по функциям.

Деревья решений регрессии - в деревьях решений такого типа переменная решения является непрерывной. 
У дерева есть корневой узел и конечные узлы. После создания корневого узла мы можем построить дерево, выполнив две части:

1. При создании терминальных узлов дерева решений одним из важных моментов является решение о том, когда прекратить выращивание дерева или создать дополнительные терминальные узлы. Это может быть сделано с использованием двух критериев, а именно: максимальная глубина дерева и минимальная запись узла следующим образом:

- Максимальная глубина дерева — как следует из названия, это максимальное количество узлов в дереве после корневого узла. Мы должны прекратить добавлять терминальные узлы, как только дерево достигнет максимальной глубины, т.е. как только дерево получит максимальное количество терминальных узлов.

- Записи минимального узла- его можно определить как минимальное количество шаблонов обучения, за которое отвечает данный узел. Мы должны прекратить добавлять терминальные узлы, когда дерево достигнет этих минимальных записей узлов или ниже этого минимума.

2. Терминальный узел используется для окончательного прогноза.

Рекурсивное расщепление - это метод построения дерева. В этом методе после создания узла мы можем рекурсивно создавать дочерние узлы (узлы, добавленные к существующему узлу) для каждой группы данных, сгенерированной путем разделения набора данных, вызывая ту же функцию снова и снова.

После построения дерева решений нам нужно сделать прогноз об этом. По сути, прогнозирование предполагает навигацию по дереву решений с помощью специально предоставленной строки данных.

Мы можем сделать прогноз с помощью рекурсивной функции, как это было сделано выше. Та же самая процедура предсказания вызывается снова с левыми или дочерними правыми узлами.

$* * *$

1. Уэс Маккинли Python и анализ данных / Пер. с англ. Слинкин А.А. - М.: ДМК Пресс, 2015, -482 c: ил.

2. Рашка C.Python и машинное обучение / пер. с англ. А.В. Логунова. - М.: ДМК Пресс, 2017. - 418 с.: ил.

3. «Открытый курс машинного обучения: анализ временных рядов с помощью Руthon [Электронный pecypc] Режим доступа: Интернет: https://habr.com/ru/company/ods/blog/327242

4. Обзор методов классификации в машинном обучении [Электронный ресурс] Режим доступа: Интернет: https://tproger.ru/translations/scikit- learn-in-python

\section{Люкин Д.В. \\ Различные подходы к решению проблемы рекомендации мультимедийного контента}

МИРЭА - Российский технологический университет (Россия, Москва)

doi: $10.18411 / \mathrm{j}-05-2021-16$

\section{Аннотация}

Мультимедийный контент достаточно плотно вошел в жизни людей. Новостные ресурсы все чаще прилагают различные изображения, аудио и видео сообщения к своим статьям, обучающие ресурсы постепенно переходят с обучения по текстовым материалам на гибридные подходы, владельцы интернет-ресурсов добавляют все больше различных типов медиа на свои сайты, чтобы удовлетворить потребности большего числа пользователей, поэтому сейчас так важно уметь правильно 